Holger Scheib • Iain McLay • Nicolas Guex •

Jeff J. Clare $\cdot$ Frank E. Blaney $\cdot$ Tim J. Dale •

Simon N. Tate $\cdot$ Graeme M. Robertson

\title{
Modeling the pore structure of voltage-gated sodium channels in closed, open, and fast-inactivated conformation reveals details of site 1 toxin and local anesthetic binding
}

Received: 16 April 2005 / Accepted: 15 September 2005 / Published online: 1 March 2006

C) Springer-Verlag 2006

\begin{abstract}
In this work molecular modeling was applied to generate homology models of the pore region of the $\mathrm{Na}_{v} 1.2$ and $\mathrm{Na}_{v} 1.8$ isoforms of human voltage-gated sodium channels. The models represent the channels in the resting, open, and fast-inactivated states. The transmembrane por-
\end{abstract}

Electronic Supplementary Material Supplementary material is available for this article at http://dx.doi.org/10.1007/s00894-00

H. Scheib $(\bowtie)$

SBC Lab AG,

Seebüelstrasse 26,

8185 Winkel, Switzerland

e-mail: holger.scheib@sbclab.com

Tel.: +41-44-8625086

Fax: +41-44-8625087

H. Scheib

Department of Structural Biology and Bioinformatics,

University of Geneva,

1 , rue Michel-Servet,

1211 Geneva 4, Switzerland

H. Scheib

Swiss Institute of Bioinformatics,

Centre Médicale Universitaire,

1 , rue Michel-Servet,

1211 Geneva 4, Switzerland

I. McLay $\cdot$ J. J. Clare $\cdot$ T. J. Dale $\cdot$ S. N. Tate

GlaxoSmithKline Medicines Research Centre,

Gunnels Wood Road,

Stevenage Hertfordshire, SG1 2NY, UK

N. Guex

GlaxoSmithKline,

Five Moore Drive,

Research Triangle Park, NC 27709, USA

F. E. Blaney

GlaxoSmithKline New Frontiers Science Park (North),

Third Avenue,

Harlow, Essex, CM19 5AW, UK

G. M. Robertson

sienabiotech $\mathrm{SpA}$,

via Fiorentina 1 ,

53100 Siena, Italy tions of the channels were based on the equivalent domains of the closed and open conformation potassium channels KcsA and MthK, respectively. The critical selectivity loops were modeled using a structural template identified by a novel 3D-search technique and subsequently merged with the transmembrane portions. The resulting draft models were used to study the differences of tetrodotoxin binding to the tetrodotoxin-sensitive $\mathrm{Na}_{v} 1.2\left(\mathrm{EC}_{50}: 0.012 \mu \mathrm{M}\right)$ and -insensitive $\mathrm{Na}_{v} 1.8\left(\mathrm{EC}_{50}: 60 \mu \mathrm{M}\right)$ isoforms, respectively. Furthermore, we investigated binding of the local anesthetic tetracaine to $\mathrm{Na}_{v} 1.8\left(\mathrm{EC}_{50}: 12.5 \mu \mathrm{M}\right)$ in resting, conducting, and fast-inactivated state. In accordance with experimental mutagenesis studies, computational docking of tetrodotoxin and tetracaine provided (1) a description of site 1 toxin and local anesthetic binding sites in voltagegated sodium channels. (2) A rationale for site 1 toxinsensitivity versus -insensitivity in atomic detail involving interactions of the $\mathrm{Na}_{v} 1.2$ residues F385-I and W943-II. (3) A working hypothesis of interactions between $\mathrm{Na}_{v} 1.8$ in different conformational states and the local anesthetic tetracaine.

Keywords Voltage-gated sodium channels . Tetrodotoxin - Tetracaine - Protein structure modeling · Computational docking

\section{Introduction}

Human voltage-gated sodium channels are large multidomain complexes comprising high sequence similarity $[1$, 2]. Their $\alpha$-subunits, which form the ion-permeation pore are responsible for ion-channel gating and the selective permeation of $\mathrm{Na}^{+}$ions. The long polypeptide chain folds into four non-identical domains (I to IV) [3], each of which is composed of six transmembrane $\alpha$-helices (S1 to S6). The permeation pore is positioned at the extracellular side of the cell membrane and is formed by S5 and S6, linked by P-loops. The latter fold partly back into the membrane to form the so-called outer vestibule. This hosts the selectivity 


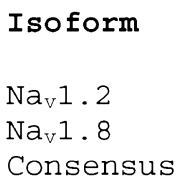

$\mathrm{Na}_{\mathrm{V}} 1.8$
Consensus

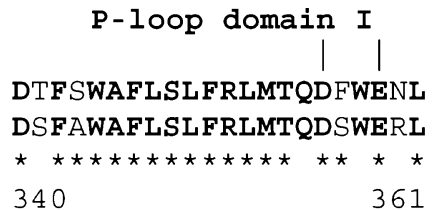

P-loop domain III

DNVGLGYLSLLQVATFKGWMDI DNVAMGYLALLQVATFKGWMDI

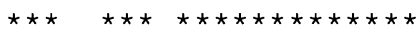

1351

Fig. 1 The P-loop sequences of human voltage-gated sodium channel isoforms $\mathrm{Na}_{v} 1.2$ and $\mathrm{Na}_{v} 1.8$. Identical residues are bold and marked by an asterisk ("*") in the consensus line. The amino acids of the outer vestibule forming the toxin binding site 1 are shown between pipes (" 1 "). The toxin binding site 1 differs in one position

filter (DEKA-motif), which comprises four different amino-acid side chains, one from each domain: aspartic acid (I), glutamic acid (II), lysine (III), and alanine (IV). The same P-loops shape the binding site for site 1 toxins, such as tetrodotoxin (TTX), involving in each domain the selectivity filter residue and its three C-terminal neighbors (Fig. 1). However, human isoforms of voltage-gated sodium channels are either sensitive or resistant to TTX block (Table 1) with a single amino-acid variation discriminating between these two channel types (Fig. 1). If the $\mathrm{C}$-terminal neighbor of the selectivity filter aspartic acid in domain I contains an aromatic side chain, the channel will be effectively blocked by TTX, whereas in the case of a serine or a cysteine the channels are less susceptible to blockade by site 1 toxins (for a review refer to [1]).

Voltage-gated sodium channels may also be obstructed by local anesthetics which, in contrast to site 1 toxins, enter the ion-permeation pore from the intracellular side of the channel and bind to residues of the so-called inner vestibule [2]. High-affinity binding of local anesthetics to sodium channels appears to occur largely, but not exclusively, to the inactivated state and involves the residues of the S6 transmembrane helices [4-12]. In the fast-inactivated state, the intracellular linker between domains III and IV acts as

Table 1 Average estimated binding energies for ligand-channel complexes, $E_{\text {ass }}$, and $\mathrm{EC}_{50}$ values for tetrodotoxin $\left(\mathrm{Na}_{v} 1.2\right.$ and $\left.\mathrm{Na}_{v} 1.8\right)$ and tetracaine $\left(\mathrm{Na}_{v} 1.8\right)$

\begin{tabular}{|c|c|c|c|c|}
\hline & $\begin{array}{l}E_{\text {ass }} \\
{\left[\mathrm{kJ} \mathrm{mol}^{-1}\right]}\end{array}$ & & & $\begin{array}{l}\mathrm{EC}_{50} \\
{[\mu \mathrm{M}]}\end{array}$ \\
\hline $\mathrm{Na}_{v} 1.2$ Tetrodotoxin & -84 & & & $0.012^{\mathrm{a}}$ \\
\hline \multirow[t]{3}{*}{$\mathrm{Na}_{v} 1.8$ Tetrodotoxin } & -97 & & & $60^{\mathrm{b}}$ \\
\hline & Closed & $\begin{array}{l}\text { Fast- } \\
\text { inactivated }\end{array}$ & Open & \\
\hline & $\begin{array}{l}E_{\text {ass }} \\
{\left[\mathrm{kJ} \mathrm{mol}^{-1}\right]}\end{array}$ & $\begin{array}{l}E_{\text {ass }} \\
{\left[\mathrm{kJ} \mathrm{mol}^{-1}\right]}\end{array}$ & $\begin{array}{l}E_{\text {ass }} \\
\quad\left[\mathrm{kJ} \mathrm{mol}{ }^{-1}\right]\end{array}$ & $\begin{array}{l}\mathrm{EC}_{50} \\
{[\mu \mathrm{M}]}\end{array}$ \\
\hline $\mathrm{Na}_{v} 1.8$ Tetracaine & $-^{c}$ & -99 & -88 & $12.5^{\mathrm{d}}$ \\
\hline
\end{tabular}

${ }^{\text {a Noda et al. [46] }}$

${ }^{\mathrm{b}}$ Akopian et al. [47]

${ }^{c}$ No valuable docking results obtained

${ }^{\mathrm{d}}$ TJD unpublished results
1372

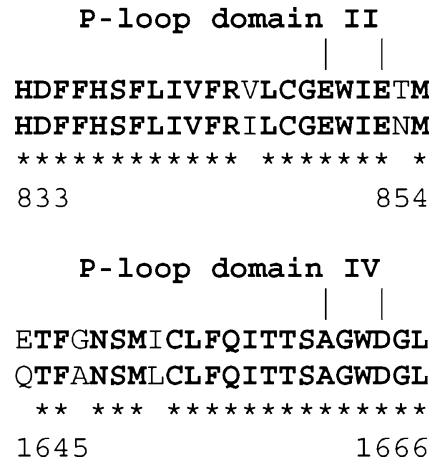

only: F385-I in $\mathrm{Na}_{v} 1.2$ and $\mathrm{S} 357-\mathrm{I}$ in $\mathrm{Na}_{v} 1.8$, respectively. The residues of the selectivity filter DEKA-locus represent the $\mathrm{N}$ terminal (left limit), the outer ring residues the $\mathrm{C}$-terminal border (right limit) of the outer vestibule

the so-called inactivation gate [13]. It has been hypothesized that this loop would fold into the open channel pore during inactivation. Experimental studies have revealed that a hydrophobic IFM-motif within this loop is essential for fast inactivation to occur [14-16].

However, to date all efforts to determine experimentally the $3 \mathrm{D}$-structure of the ion-permeation pore of voltagegated sodium channels have failed. This prevents the many experimental results for these channels being put into a wider context to reveal interaction networks between residues in spatial proximity to one other. One way to approach this problem is to model the structure of a voltage-gated sodium channel based on an experimental structure for a related membrane-bound protein. Yet early modeling efforts were hampered by the fact that no experimental structures were available to guide these experiments. Hence, modeling concentrated mainly on identifying P-loop architectures that corresponded to functional and mutagenesis data. Lipkind, Fozzard, and coworkers published a model for the toxin binding site 1 of the mammalian $\mathrm{Na}_{v} 1.4$ isoform in which the P-loops were $\beta$-hairpins $[17,18]$. In contrast, Guy and Durell postulated that the P-loops in sodium channels would form a $\alpha$-helixturn motif, followed by an extended segment $[19,20]$. The first X-ray structures for KcsA and MthK [21-23] revealed that the overall topology of the P-loops in potassium channels was indeed similar to the one proposed by the Guy and Durell model. But it remained unclear whether or not the different selectivity filters in sodium and potassium channels required a different P-loop topology as well. In their most recent model, Lipkind and Fozzard postulated that the P-loops in voltage-gated sodium channels adopted the substructure of a $\alpha \beta$-arch, a $\alpha$-helix-turn- $\beta$-strand, rather than a $\alpha$-helix-turn-coil motif [24]. In order to include as many experimental data as possible, i.e. interactions with the site 1 toxins tetrodotoxin and saxitoxin, and to satisfy structural constraints imposed from the KcsA template, their P-loops were placed differently with respect to KcsA. Their toxin binding site was wider outside and narrowed toward the selectivity filter. Still, in their model the $\mathrm{C} \alpha$ atoms of the selectivity filter locus were further apart from the axis of $\mathrm{Na}^{+}$ 
permeation than their corresponding partners in the potassium channel structures. Nevertheless, the Lipkind and Fozzard model allowed explaining both the access of tetrodotoxin and saxitoxin to the DEKA-locus as well as favorable interactions of these ligands with residues of the so-called outer ring (EEMD-motif in position +3 of the DEKA-locus, (Fig. 1) [24, 25]. Furthermore, their model provided evidence for the permeation of larger organic cations through sodium as compared to potassium channels [25]. Recently, Tikhonov and Zhorov suggested a model for the outer vestibule of $\mathrm{Na}_{v} 1.4$ by shaping the binding site around a rigid toxin ligand. The starting configuration of the P-loops was as in the open conformation potassium channel MthK [26]. The authors assumed strong structural similarity between sodium and potassium channels, however, their model allowed retracing important interactions with the site 1 toxins tetrodotoxin, saxitoxin, and $\mu$ conotoxin, respectively.

Despite these modeling efforts, only little is known about the structural implications of site 1 toxin binding to different sodium-channel isoforms, i.e. TTX-sensitive and TTX-insensitive isoforms. This is even more the case for modeling interactions of voltage-gated sodium channels with local anesthetics. To our knowledge, a systematic structural analysis of the interactions between local anesthetics and voltage-gated sodium channels considering the structural dynamics of the channel protein has not been carried out to date. To address these open questions, we applied molecular modeling to generate model structures of the pore regions of $\mathrm{Na}_{v} 1.2$ and $\mathrm{Na}_{v} 1.8$ in different conformational and thus functional states. Our models contain the binding sites for both site 1 toxins and local anesthetics. In a first step, we computationally docked tetrodotoxin to the TTX-sensitive $\mathrm{Na}_{v} 1.2$ and the TTXresistant $\mathrm{Na}_{v} 1.8$ isoforms and investigated the interactions between the ligand and the receptor. Although the outer vestibules of $\mathrm{Na}_{v} 1.2$ and $\mathrm{Na}_{v} 1.8$ differ in one amino acid only (Fig. 1), this subtle difference suffices to affect their binding activity toward TTX by ca. 5,000-fold (Table 1). Similarly, the local anesthetic binding sites of the two isoforms are almost identical but significant differences in affinity toward tetracaine have not been reported so far. Not surprisingly, computational docking of tetracaine to $\mathrm{Na}_{v} 1.2$ and $\mathrm{Na}_{v} 1.8$ in closed, open, and fast-inactivated conformation yielded similar results.

\section{Materials and methods}

\section{Modeling transmembrane segments}

Using the Swiss PDB Viewer (SPDBV) environment [27] the S5 and $\mathrm{S} 6$ transmembrane segments of $\mathrm{Na}_{v} 1.2$ (SwissProt accession Q99250) and $\mathrm{Na}_{v} 1.8$ (TrEMBL accession Q9Y5Y9) were aligned to the corresponding M1 and M2 helices of KcsA (PDB code 1BL8 [23]) and MthK (PDB code $1 \mathrm{LNQ}[21,22]$ ), respectively (Fig. 2). Alignment of the S5 sequences onto M1 of KcsA and MthK was anchored at a conserved glycine residue four positions upstream of the C-terminal helix end. Also, in MthK and almost all isoforms of sodium channels, a phenylalanine is located at the C-terminus of M1 and S5, respectively. The structural alignment of the S6 TM-helices was anchored at a functionally important glycine residue for channel opening, G99 in KcsA and G83 in MthK [21]. Additionally, reported interactions between local anesthetics and residues of various mammalian sodium channel isoforms, revealed by site-directed mutagenesis [4-12], were used to align these residues such that they faced the ion permeation pore wherever experimental data suggested so. For domain II, no such interactions have been reported to date. Therefore, the target to template alignment for S6-II was anchored exclusively on the putative glycine hinge residue G970 in $\mathrm{Na}_{v} 1.2$ and G877 in $\mathrm{Na}_{v} 1.8$ aligned with G99 of KcsA and G83 of MthK, respectively. From the resulting structural alignment model structures were generated for four unconnected S5 and S6 helices each using the homology modeling engine promodII [27].

\section{Modeling P-loops}

A suitable template structure for the P-loops was found by searching a representative non-redundant set of 474 PDBfiles. The structures shared less than $35 \%$ sequence identity and no fold similarity. The search query was programmed applying a modified version of the SPDBV scripting language. A search was performed to select fragments that could be subsequently grafted onto the anchor residues of the transmembrane helix bundle. To allow for adequate search flexibility fragments were required to contain 12 to 16 residues of $\alpha$-helix secondary structure followed by either three to 13 coiled residues or three to seven coiled residues and three to six residues of $\beta$-strand secondary structure. The identified fragments were clustered within SPDBV according to their rmsd value compared to the first hit of the search. Each time the rmsd to any previously defined cluster was above $2.0 \AA$ a new cluster was created. Representatives of each cluster were then inspected manually for possible fitting onto the transmembrane helix bundle, which was determined predominantly by sterics. In order to fit onto the transmembrane helices the loops were required to turn sharply between the N-terminal $\alpha$-helix and the C-terminus. Further selection criteria were the overall space occupied by the loop, the orientation of side chains, i.e. in the turn and C-terminus, and the enthalpy of the resulting structure. In this way N523 to Y544 of glutamine amidotransferase from E. coli (PDB code $1 \mathrm{MOQ}$ [28]) was chosen as the best modeling template. The 12 residues from N523 to E534 are $\alpha$-helical, followed by ten residues in coiled conformation from E535 to Y544. The sequence fragments of the P-loops were aligned on the respective fragment of the $1 \mathrm{MOQ}$ template and homology models built using promodII [27]. As a result, four P-loop model structures, one for each domain, were obtained. To validate our choice of P-loop template, the template-selection step was carried out using transmembrane helix bundles from closed and open conforma- 
Fig. 2 Domain-separated multiple sequence alignment of the S5 and S6 helices of human $\mathrm{Na}_{v} 1.2$ and $\mathrm{Na}_{v} 1.8$ to the M1 and M2 transmembrane helices of the KcsA and MthK templates. Identical residues between the two sodium channel isoforms are bold and marked by an asterisk ("*") in the consensus line. Sequence numbering is according to $\mathrm{Na}_{v} 1.8$. Filled squares indicate residues involved in sodium channel activation. Open squares highlight positions that were experimentally found to interact with local anesthetics. In our models, residues aligned to black dots form the local anesthetic binding site. Open circles represent the positions of the gating hinge glycine residues in MthK as found by Jiang et al. [21, 22]

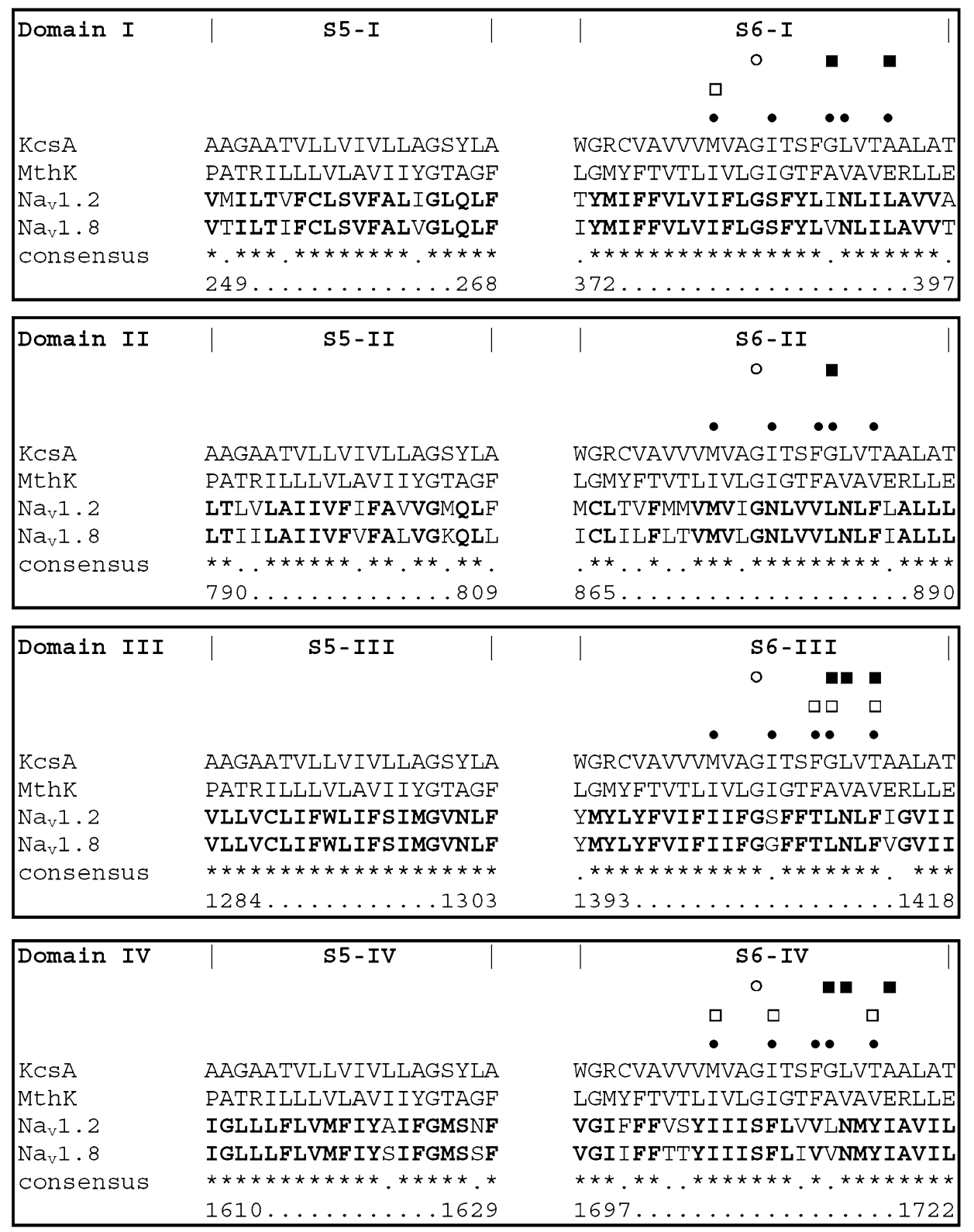

tion models. It was found that the extracellular region of the transmembrane helices and P-loops vary only slightly in the two conformations and that major rearrangements occur predominantly in the cytosolic part. As a consequence, these minor structural variations in the P-loop region did not affect our choice of template (data not shown).

\section{Assembling fragments}

The four P-loop models were placed manually on top of the transmembrane helices guided by the positions of both these helices and the P-loops in KcsA and MthK, respectively (Fig. 3). Steric clashes between fragments were removed through manual manipulation and the enthalpy of the model structures was minimized during two by 200 steps of Steepest Descent simulations using the GROMOS96 force field [29] as implemented in SPDBV [27].

Fast-inactivated form The inactivation gate substructure was placed manually to the open state model using the NMR-structure of a 21-residue fragment of the rat brain channel II (PDB code 1BYY [30]). Its position in the structure reflects the most prominent interactions of inactivation gate residues, i.e. I1433 and F1434, as well as residues of S6-IV, i.e. F1710 and Y1717 (numbering according to $\mathrm{Na}_{v} 1.8$ ). Potential clashes between residues were identified in SPDBV and removed applying energy minimization as described in the general protocol. 

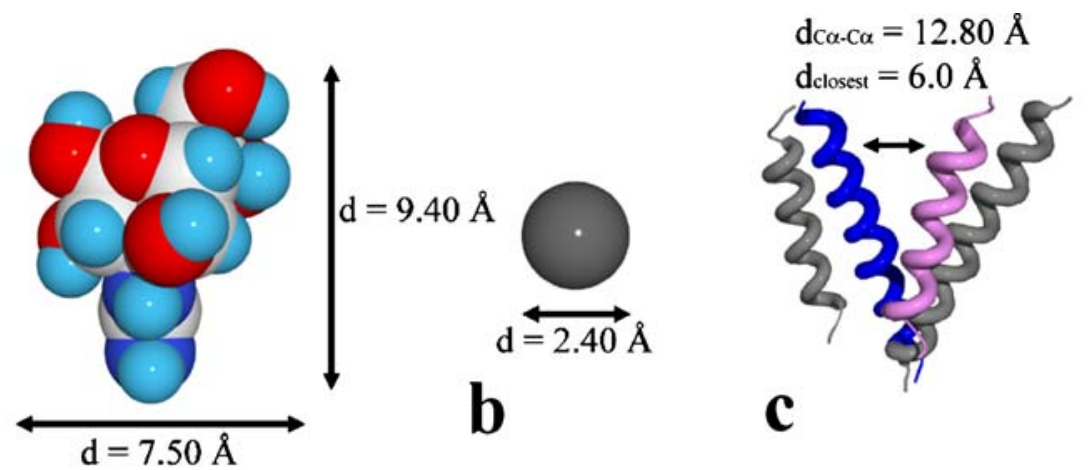

a
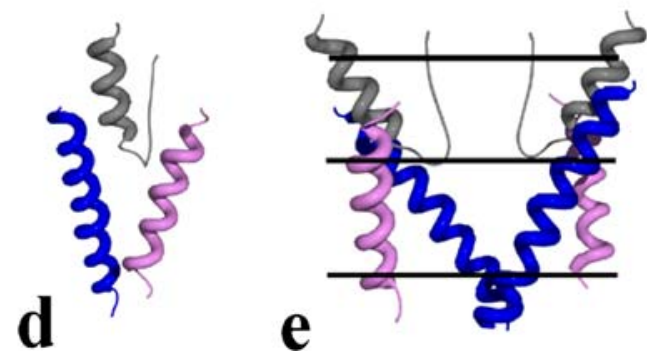

Fig. 3 Modeling the assembly of P-loops and transmembrane part of $\mathrm{Na}_{v} 1.2$ and $\mathrm{Na}_{v} 1.8$ was based on geometrical restraints. (a) Maximum expansion of tetrodotoxin. The distance $d=7.50 \AA$ represents the distance between the two hydroxyl groups of $\mathrm{C} 4$ and C10. $d=9.40 \AA$ is the distance between $\mathrm{NH}$ of the 1,2,3guanidinium group and the C11 hydroxyl [48]. Tetrodotoxin is in CPK representation and colored by atom type. (b) Diameter of an octahedrally coordinated $\mathrm{Na}^{+}$ion [49]. Although the exact coordination number of $\mathrm{Na}^{+}$in the selectivity filter of voltagegated sodium channels remains unclear, a six-fold octahedral coordination was assumed following the work of Allen et al. [50]. (c) Closest $\mathrm{C} \alpha-\mathrm{C} \alpha$ and overall distance between S5-I

\section{Structure validation}

To validate the quality of the resulting model structures, a two-fold strategy was followed. Firstly, the models were investigated for structural errors. Within the SPDBV environment, checks for clashes between residues were performed and potentially high enthalpy values detected [27]. In addition, PROCHECK [31] was used for a more detailed assessment of the model quality. Secondly, the resulting model structures were required to satisfy available biochemical knowledge, i.e. functional information, mutagenesis data, and interactions with site 1 toxins and local anesthetics.

Docking of site 1 toxins and local anesthetics

Computational docking of tetrodotoxin and tetracaine was performed using the FLO software [32], which allows for flexibility in both the ligand and the protein. The region including the selectivity filter and the outer and inner vestibule was used as the docking target and free movement around the residues lining these pockets was allowed. For each of the ligands, 500 Monte Carlo searches were performed applying a FLO force field modified to reproduce correctly the torsional preferences for the tetracaine (pink) and S6-II (blue). S6-I and S5-II are also displayed and colored in gray. This restraint limits the expansion of the P-loop substructure as well as its orientation when placed onto the transmembrane helices. (d) Position of the P-loop between S5-I (pink) and S6-II (blue). (e) The models contain two ligand binding sites. The binding site for site 1 toxins is located extracellular (top) to the selectivity filter (central plane), whereas the local anesthetic binding site is toward the cytosol (bottom). The top and bottom planes sketch the limits of both binding sites. For reasons of simplicity, the channel model is represented by two opposite domains with the S5-helices in pink, S6 in blue, and the P-loops in gray

di-ortho aryl-amide bond (orthogonal). The ten best scoring results from FLO were selected and investigated further.

\section{Results}

Defining restraints to model the toxin binding site 1

Prior to the model-generation step, biochemical and mutagenesis data were collected for $\mathrm{Na}_{v} 1.2, \mathrm{Na}_{v} 1.8$ and other isoforms of mammalian voltage-gated sodium channels. In an approach similar to pharmacophore modeling, these data were translated into geometrical and physico-chemical constraints, which the resulting models had to satisfy (Fig. 3). Firstly, the dimensions of tetrodotoxin (max. $7.5 \AA$ along and $9.4 \AA$ perpendicular to the membrane axis; Fig. 3a) and its interactions with the channel protein [17-20, 24, 26, 33] determined the shape and size of the outer vestibule. From this it was concluded that the selectivity filter and outer ring residues in the same domain had to be separated by ca. 9-10 $\AA$ ( $\mathrm{C} \alpha$ distance). Accordingly, opposite residues of the outer ring EEMDlocus (Fig. 1) were expected to be ca. $10-12 \AA$ apart $(\mathrm{C} \alpha$ distance ca. 15.8 $\AA$ ) (see below). Secondly, at its narrowest point the selectivity filter ought to allow dehydrated $\mathrm{Na}^{+}$to pass. Therefore, the ionic radius of $\mathrm{Na}^{+}$determined the minimal distance between the side chains of the DEKA- 
locus (Fig. 3b) leading to a $\mathrm{C} \alpha$ distance of opposite DEKA residues of ca. $10.5 \AA$. Thirdly, the most membraneoriented selectivity filter residues T75 and T59 in KcsA and MthK, respectively, determined how deep the sodium channel P-loops would fold back into the membrane, since the residues of the DEKA-locus were structurally aligned to them. As a consequence, the TTX binding site was to adopt a slightly confined shape, being ca. $5 \AA$ wider at the outer ring ( $\mathrm{C} \alpha$-distances) than at the selectivity filter (Fig. 3e). Finally, in the assembled structure, each P-loop fits into a cleft between S5 and S6 of neighboring domains (Fig. 3d) [21-24]. The closest C $\alpha$ contacts between these helices were ca. 12-13 $\AA$, which impaired what shape and horizontal extension any potential P-loop might adopt (Fig. 3c). Moreover, this cleft played an important role in placing the P-loops within the structural ensemble, as it determines the width of the $\mathrm{Na}^{+}$permeation pore at the DEKA-locus within the structural ensemble.

Modeling the toxin binding site 1 in $\mathrm{Na}_{v} 1.8$ and $\mathrm{Na}_{v} 1.2$

Numerous mutagenesis experiments revealed that the selectivity filter differs among sodium and potassium channels [2, 34-41]. Potassium channels are homo-tetrameric and their selectivity filter is formed by the backbone carbonyl oxygens of four identical TVGY repeats. In contrast, in the hetero-tetrameric voltage-gated sodium channels the side chain of only one residue per domain contributes to the selectivity filter (DEKA-motif). In order to make room for these side chains the $C \alpha$-atoms of the DEKA-locus residues were placed farther apart compared to the potassium channel templates. But, if the size of the $\mathrm{Na}^{+}$permeation pore at the DEKA-locus increases the less space will be available for the P-loops without interfering with the transmembrane helices. By comparing the experimental KcsA (closed) and MthK (open) potassium channel structures it was found that these channels undergo substantial conformational rearrangement in the cytosolic region, but not in vicinity to the P-loops leading to the conclusion that the pore structure close to the P-loops in potassium and thus most likely also in sodium channels is well conserved. Therefore, we systematically searched the known structure space for potential P-loop templates that would allow a widening of the pore at the DEKA-locus while still fitting into the space provided by the rather invariant transmembrane helices. It is generally accepted that the N-terminal part of sodium channel P-loops ought to be $\alpha$-helical $[19,20,24,26]$. Whereas this restricted our search at the N-terminus, we remained flexible with respect to the C-terminal part. Interestingly, the most suitable Ploop template was similar to the one proposed by Guy and Durell, an $\alpha$-helix followed by a turn and an extended region $[19,20]$. In this fragment the $\alpha$-helix was followed by a sharp turn which in our models allowed indeed the toxin binding site to widen toward the outer ring, despite an increased $\mathrm{C} \alpha$ distance of the selectivity filter residues. The presence of the side chains of DEKA, however, would impair that the gap for $\mathrm{Na}^{+}$to pass may not be larger than ca. $2.5 \AA$ times $2.5 \AA$, after displacement of i.e. the large lysine side chain.

In voltage-gated sodium channels in position +2 of the DEKA-locus a conserved tryptophan is found in domains I, III, and IV. Yet in domain II, this tryptophan is in position +1 (Fig. 1). In our models the side chains of the tryptophans in domains I, III, and IV pointed toward the P-loop $\alpha$-helix of their clockwise neighbor domain (II, IV and I, respectively) delimiting the outer vestibule and therefore the toxin binding site. In domain II the tryptophan was oriented toward the P-loop of domain I (counterclockwise) and was more exposed toward a potential ligand. In absence of a tryptophan in position +2 of domain II, the outer vestibule between domains II and III was confined by an isoleucine in position +2 of domain II and a methionine in +3 of domain III.

\section{Docking of site 1 toxins to $\mathrm{Na}_{v} 1.2$ and $\mathrm{Na}_{v} 1.8$}

It is well known that mutating the C-terminal neighbor of the domain I selectivity filter aspartic acid results in a 5000fold difference in binding affinity of the TTX-sensitive $\mathrm{Na}_{v} 1.2$ and the TTX-insensitive $\mathrm{Na}_{v} 1.8$ isoforms to tetrodotoxin (Table 1). To determine the mode of interaction with either isoform TTX was computationally docked to $\mathrm{Na}_{v} 1.2$ and $\mathrm{Na}_{v} 1.8$ allowing flexibility in both the ligand and the protein. In both cases attractive electrostatic interactions were observed due to the formation of a salt bridge of the 1,2,3-guanidinium group of tetrodotoxin to the selectivity filter aspartic and glutamic acid in domain I and II. Moreover, the position of the ligand in the binding pocket was further stabilized by forming hydrogen bonds to the outer ring residues, i.e. E387-I in $\mathrm{Na}_{v} 1.2$ (E359-I in $\mathrm{Na}_{v} 1.8$ ), E945-II (E852-II), and D1717-IV (D1664-IV). These interactions corresponded to earlier findings by other groups [24-26] but were insufficient to explain the much lower $\mathrm{EC}_{50}$ value of $\mathrm{Na}_{v} 1.2$ toward TTX. However, when steric interactions were considered a striking difference in ligand binding to the two isoforms could be observed. For $\mathrm{Na}_{v} 1.2$, steric interactions of the ligand with (hydrophobic) binding site residues were predominantly through contacts with F385-I and W943-II. The side chains of both residues formed a "V" to which the 1,2,3-guanidinium group of TTX fit perfectly, besides its interaction with the selectivity filter and outer ring residues (Fig. 4). This interaction may not only be steric, but may also include a cation- $\pi$ interaction between the guanidinium group and the aromatic moieties of either W943-II or F385-I [42]. As a consequence, for $\mathrm{Na}_{v} 1.2$ only one binding conformation of TTX with limited translational freedom in the binding site was observed. In contrast, in $\mathrm{Na}_{v} 1.8$ this "V"-shaped substructure was not found. Instead, the serine 357-I side chain left a void next to W850-II (Fig. 4) and the docked ligand appeared much more flexible in the binding site corresponding with a lower binding energy, $E_{\text {ass }}$ (Table 1). 


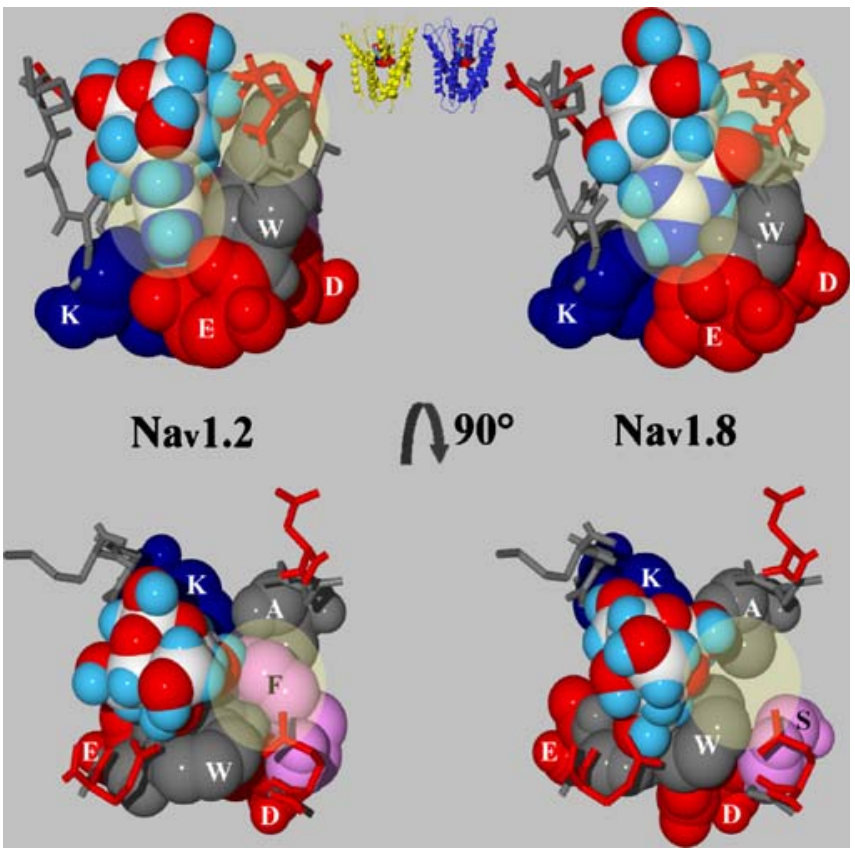

Fig. 4 Interaction of tetrodotoxin with the TTX-sensitive voltagegated sodium channel isoform $\mathrm{Na}_{v} 1.2$ (left) and the TTX-insensitive $\mathrm{Na}_{v} 1.8$ (right) seen along (top) and perpendicular (bottom) to the membrane axis. The DEKA-filter residues as well as W943-II in $\mathrm{Na}_{v} 1.2$ and W850-II in $\mathrm{Na}_{v} 1.8$ are represented in CPK and colored according to type: blue $=$ basic, red $=$ acidic, gray=hydrophobic. F385I in $\mathrm{Na}_{v} 1.2$ and $\mathrm{S} 357-\mathrm{I}$ in $\mathrm{Na}_{v} 1.8$ are shown in CPK and are colored pink. Yellow circles indicate the regions where the positions of the ligand in the binding site are most different. Top center: position of the site 1 toxin binding site in the structural models as seen along the axis of the membrane. Top left: the selectivity filter A1714-IV as well as F385-I are hidden behind the ligand. Yellow circles indicate the orientation of the guanidinium group of the ligand as well as the position of W943-II in the binding site. Top right: the selectivity filter A1661-IV and S357-I are located behind the ligand. The guanidinium group of TTX has rotated ca. $90^{\circ}$ in the binding site due to the changed position of W850-II. Bottom left: the yellow circle highlights the "V"-shape between F385-I and W943-II to which tetrodotoxin closely fits. Bottom right: The side chain of W850-II is in a different position than in $\mathrm{Na}_{v} 1.2$

\section{Local-anesthetic binding site}

The local-anesthetic binding site of voltage-gated sodium channels is located intracellular of the selectivity filter in the transmembrane part of the channel. The selectivity filter residues (DEKA) and their N-terminal neighbors (QGFS) form the extracellular limit of this binding site and prevent local anesthetics from diffusing through the membrane. From the selectivity filter, the local anesthetic binding site extends through the membrane and is composed of residues of the transmembrane helices S6, i.e. the residues that face the pore at the four helix turns that follow the selectivity filter intracellularly (Fig. 2). At the third helix turn, the side chains of two rather than one amino acid face the pore in all four domains. Altogether, the local anesthetic binding site consists of 28 highly conserved amino acids (see Electronic Supplementary Material). Sequence variation of the pore lining residues in the two isoforms was rare and subtle (I417-I, S1461-III and L1768-IV in $\mathrm{Na}_{v} 1.2$ vs. V389-I,
G1406-III and V1714 in $\mathrm{Na}_{v} 1.8$ ) (Fig. 2). Interestingly, in the position of the hinge residue in domain IV is a serine in both isoforms compared to a glycine in domains I, II and III and the potassium channel structures KcsA and MthK.

\section{Docking of local anesthetics}

Local anesthetics interact preferably with voltage-gated sodium channels in the fast-inactivated state [43]. However, they can also act on the active state and may even be trapped in the closed form [44]. In order to study the molecular basis of these observations tetracaine was docked to the local anesthetic binding site of $\mathrm{Na}_{v} 1.8$ in closed, open, and fast-inactivated conformation. It was observed that the charged amine of the ligand formed hydrogen bonds with the selectivity filter residues aspartic and glutamic acid of domain I and II, whereas the aromatic moiety of the ligand pointed toward the cytosol (Fig. 5). Whereas binding of the polar head group to the selectivity filter remained unaffected by the conformational state of the channel interactions of the channel protein with the aromatic moiety varied greatly.

Docking of the rather large tetracaine to $\mathrm{Na}_{v} 1.8$ in the closed conformation was highly unfavorable. In particular, the local anesthetic binding site narrowed toward the cytosol leaving not enough space to host the voluminous substituted aromatic part of the ligand. As a consequence, docking artifacts were observed where the ligand adopted an alternative conformation (Table 1). In this case, the aromatic moiety tended to leave the inner vestibule through an opening between the S6-helices of neighboring domains

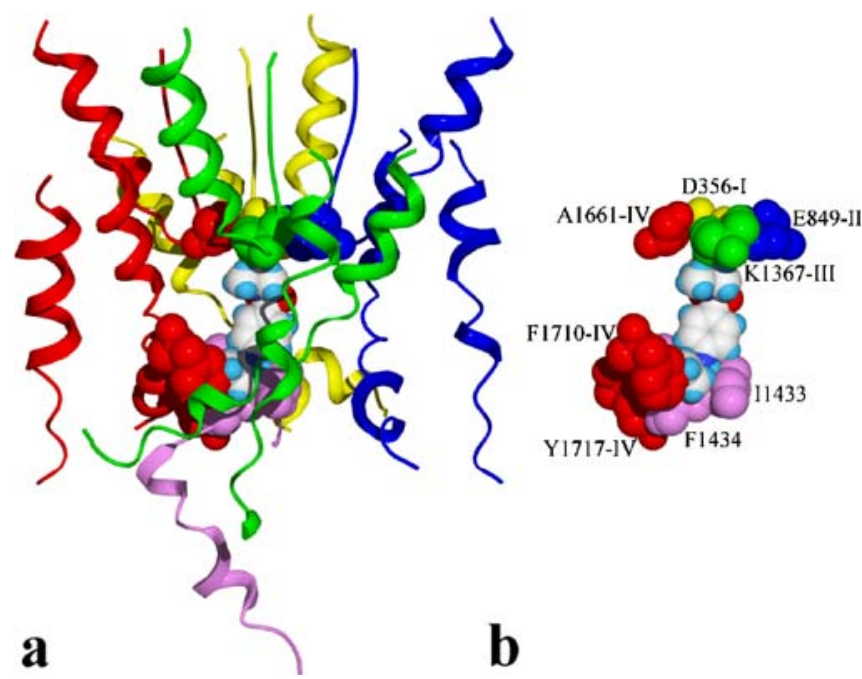

Fig. 5 Tetracaine in complex with $\mathrm{Na}_{v} 1.8$ in fast-inactivated form. The ligand is represented in CPK and colored by atom type. Ribbons and amino acids are colored by domain: yellow=domain I, blue=domain II, green=domain III, red=domain IV, pink=inactivation gate. Main interaction partners are shown in CPK. (a) Tetracaine bound to the inner vestibule. View along the membrane plane. (b) Same view as in a but limited to main interaction partners only. The polar head group of tetracaine interacts with the DEKA-motif residues, its hydrophobic tail with the hydrophobic and mainly aromatic residues of S6-IV and the inactivation gate 
in the direction of the membrane. However, the binding site is large enough to trap smaller organic molecules and even small local anesthetic drugs (data not shown). In contrast, tetracaine docking to the open and in particular to the fastinactivated form yielded substantially better energy values. As for the closed form, the charged amino group formed hydrogen bonds to either aspartic or glutamic acid of the selectivity filter. But in the models representing the fastinactivated form the inactivation gate structure blocked access to the inner vestibule and exposed the conserved IFM-motif to the ligand (Fig. 5). Hence, tetracaine was surrounded entirely by residues of the sodium channel and, as a consequence, ligand movement within the binding site was substantially confined. Docking of tetracaine to the fast-inactivated form was energetically most favorable (Table 1). It is suggested that the energy difference compared to docking to the open conformation model was due to the presence of interactions which trapped the ligand sterically in a predominantly hydrophobic binding site. Common main interactions of the ligand occurred mainly with F1710-IV and the inactivation gate residue F1434 and to a lesser extend with Y1717-IV and I1433 corresponding to interactions reported for local anesthetics with other sodium channel isoforms $[11,12]$. Furthermore, tetracaine formed a H-bond to the conserved N1411 in the third helix turn of S6-III. We postulate that in the presence of tetracaine water may be largely excluded from the inner vestibule of the fast-inactivated channel. However, we consider it possible that a few water molecules may be present close to the selectivity filter where interactions between the ligand and the channel are predominantly polar. Additionally, the opening between the S6-helices may host several water molecules.

\section{Discussion}

What does the outer vestibule look like?

It has long been assumed that the different nature and composition of the selectivity filters in sodium and potassium channels would have resulted in differently shaped 3D structures around the selectivity filter [1]. In the absence of experimental structures for voltage-gated sodium channels it was attempted to predict the structure of the outer vestibule including the selectivity filter $[17-20,24$, $26,33]$. However, the resulting models were not unambiguous with respect to the topology of the individual P-loops and with respect to how the four P-loops assembled to form the toxin binding site 1 . It is accepted that, as in potassium channels, the N-terminal part of the sodium channel Ploops is $\alpha$-helical [19, 20, 24, 26]. Yet these models differ in the secondary structure following the $\alpha$-helix-turn. Lipkind and Fozzard believed that the $\alpha$-helix-turn was followed by a $\beta$-strand ( $\alpha \beta$-arch) [24], whereas Guy and Durell suggested several residues in coil conformation [19, 20]. However, both groups postulated that the backbone atoms in the sodium channel P-loops had to be further apart from each other than in potassium channels to make room for the side chains of the selectivity filter residues. This displacement would lead to clashes between residues of the S5 and S6 transmembrane helices and the P-loop. In order to avoid such unfavorable interactions, the P-loops in the Lipkind and Fozzard model were twisted compared to the KcsA template. Nevertheless, both models allowed rationalizing mutagenesis data and retracing important interactions of voltage-gated sodium channels with i.e. tetrodotoxin and saxitoxin $[19,20,25]$. Recently, Tikhonov and Zhorov built models for the P-loops of $\mathrm{Na}_{v} 1.4$ by shaping the binding site around rigid molecules of tetrodotoxin, saxitoxin and $\mu$-conotoxin by means of Monte-Carlo minimization [26]. Their approach was interesting, since in the starting conformation the P-loops were placed as in the MthK structure. In their models, the solution structures for the outer vestibule were highly similar and thus independent from the simulated toxin. This indicates that the binding site for at least smaller site 1 toxins, such as tetrodotoxin and saxitoxin, is rather rigid. Moreover, it is common to all models that the toxin binding site of voltage-gated sodium channels is most likely to be confined at the DEKA-locus and opens toward the outer ring $[19,20,24,26]$. Evidently, interactions with toxins require that key amino acids be placed at specific positions in space, allowing for certain flexibility in the topology and the arrangement of the P-loops to one another.

One open question remains the order of secondary structure for the amino acids following the turn at the selectivity filter. Our models and earlier work give evidence that this may be of no higher order $[19,20,26]$. This is supported by the fact that the positions of the Cterminal part of the P-loops were affected during MonteCarlo simulations [26]. Higher order secondary structure might either limit such displacement or would break down in order to preserve favorable interactions with the ligand. Additionally, in this work a systematic search of the PDB for suitable fragments yielded as the best result a $\alpha$-helixturn motif succeeded by residues in coiled conformation.

A second open question addresses the position of the DEKA-locus with respect to the potassium-channel structures. Tikhonov and Zhorov postulated a minimally wider pore at the selectivity filter compared to the potassium channels, whereas in our models the P-loops were even more open at the DEKA-locus. From an evolutionary viewpoint it might be advantageous if only one selectivity filter architecture had evolved and existed with minor structural and thus functional modification in several cation channel families. However, the more similar the DEKA-locus to the selectivity filter of potassium channels, the more difficult the correct orientation of the side chains. In the model by Tikhonov and Zhorov, the side chains of the selectivity filter aspartic acid and alanine do not face the pore due to sterical restrictions. On the contrary, in our models the side chains of all four DEKA residues faced the pore, but the wider pore made it necessary to rearrange the positions of the P-loops in order to avoid clashes with the transmembrane helices.

Our models for $\mathrm{Na}_{v} 1.2$ and $\mathrm{Na}_{v} 1.8$ were accurate enough to comprehend biochemical and mutagenesis data and to 
study isoform-specific interactions with tetrodotoxin for the first time (see below).

\section{Sensitivity versus insensitivity toward site 1 toxins}

Varying the C-terminal neighbor of the selectivity filter aspartic acid in domain I determines whether a voltagegated sodium channel is sensitive or insensitive to block by tetrodotoxin $[2,34,35-41]$. In this work, computational docking of TTX to the TTX-sensitive $\mathrm{Na}_{v} 1.2$ and TTX-insensitive $\mathrm{Na}_{v} 1.8$ sodium channel isoforms was performed. Besides the known attractive electrostatic interactions between the 1,2,3-guanidinium group and the hydroxyl groups of TTX and the acidic residues of the selectivity filter and the outer ring, respectively [17, 24-26], a steric component was discovered including the $\mathrm{C}$-terminal neighbor of the DEKA-motif aspartic acid and a conserved tryptophan of domain II. Remarkably, in domains I, III, and IV a tryptophan is conserved in position +2 of the selectivity filter. In our models, the side chains of these residues were oriented toward the $\alpha$-helix of the same domain capping the gap between the P-loops of domains II, IV, and I, respectively. In domain II, the conserved tryptophan is in position +1 of the selectivity filter glutamate and its side chain is oriented toward the domain I rather than the domain III P-loop. As a result, the outer vestibule between domains II and III was not limited by a tryptophan but by the isoleucine in position +2 of domain II and by the methionine in position +3 of domain III of the respective selectivity filter residues. It is worth noting that the outer vestibule and thus the binding site for type 1 toxins is asymmetric setting voltage-gated sodium channels apart from the four-fold symmetric potassium channels. In $\mathrm{Na}_{v} 1.2$, the side chains of W943-II and F385-I came close to each other forming a "V" to which the 1,2,3-guanidinium group of TTX could fit tightly (Fig. 4). Observed interactions of both residues with the ligand were predominantly steric, but we postulate also cation- $\pi$ interactions between the 1,2,3-guanidinium group and the aromatic side chains [42]. In $\mathrm{Na}_{v} 1.8$, the "V"-pocket did not exist. Here, the smaller and more flexible serine side chain in position 357-I created a void compared to $\mathrm{Na}_{v} 1.2$ and allowed for increased flexibility of both the ligand and the protein in this region (Fig. 4). We strongly assume that the observed interactions do apply for other isoforms as well. Therefore, effective binding of TTX and other site 1 toxins (data not shown) would only occur when the aromatic neighbor of the domain I selectivity filter residue interacted closely with the tryptophan in domain II. This aromatic residue may either be a phenylalanine $\left(\mathrm{Na}_{v} 1.1, \mathrm{Na}_{v} 1.2\right)$ or a tyrosine $\left(\mathrm{Na}_{v} 1.3, \mathrm{Na}_{v} 1.4, \mathrm{Na}_{v} 1.6, \mathrm{Na}_{v} 1.7\right)$. In the case of a tyrosine we do not rule out the formation of an additional hydrogen bond between the hydroxyl group of the tyrosine and the ligand (data not shown). In contrast, if this residue was aliphatic (cysteine in $\mathrm{Na}_{v} 1.5$, serine in $\mathrm{Na}_{v} 1.8$ and $\mathrm{Na}_{v} 1.9$ ) the sterical component of interaction with the ligand was less pronounced and binding less unambiguous resulting in a significantly lower binding affinity.
Binding of local anesthetics to $\mathrm{Na}_{v} 1.8$ in different conformational states

This work represents the first attempt to investigate in silico the binding of local anesthetics to a voltage-gated sodium channel in different conformational states. We chose tetracaine as a test compound, because the binding affinity of $\mathrm{Na}_{v} 1.8$ toward tetracaine was in the same low $\mu$ molar range (Table 1), common to many local anesthetics (http:// www.iuphar-db.org/iuphar-ic/sodium.html). Our docking experiments revealed that tetracaine interacted most favorably with the open conformation channel, which was blocked by the inactivation gate. Upon occlusion of the local anesthetic binding site by the inactivation gate tetracaine was trapped inside the channel leaving its hydrophobic moiety exposed to a predominantly hydrophobic environment. Besides hydrogen bonding of the polar head group with D356-I and E849-II of the selectivity filter, main interactions occurred with the S6-IV helix residues F1710 and Y1717 as well as with I1433 and F1434 of the inactivation gate, residues which were described earlier as being essential for local anesthetics binding (for an overview [11, 12]). The ligand was additionally stabilized in this position by forming an $\mathrm{H}$ bond to N1411-III, a residue found to affect the binding of local anesthetics in rat $\mathrm{Na}_{v} 1.2$ [12]. When docked to the open conformation model, the polar interactions between the tetracaine head group and the DEKA-locus were preserved. However, the non-polar aromatic moiety was less tightly bound to the local anesthetic binding site and in vitro would have, at least partly, been exposed to water. Our docking studies also indicated that the local anesthetic binding site might be large enough to allow binding of smaller drugs, such as lidocaine and etidocaine, to the closed channel as well (data not shown). In this case it might be possible that local anesthetics with higher binding affinity might not be released from the binding site when the channel transforms from the fast-inactivated to the resting state as suggested by Vedantham and Cannon [44]. On the contrary, the size and chemical nature of tetracaine may not be exceeded without affecting the position of the inactivation gate and thus the closure of the ion permeation pore. If the ligand was larger or more rigid, i.e. by substitutions in the aromatic moiety, the inactivation gate might not be able to close, at least not completely. This hypothesis is in line with earlier observations for larger local anesthetic drugs, which block ion permeation in the open but not in the fast-inactivated state (for an overview [45]).

Limitations of the models

The models presented here are useful working models to study the principles of sodium channel gating and biochemical function. In the absence of experimental 3Dstructures such draft models are powerful tools to rationalize and plan mutagenesis experiments (including disease-associated single amino acid polymorphisms) and 
biochemical studies of voltage-gated sodium channels. However, the accuracy of these models is inferior to that of experimentally determined structures, affecting the interpretation of structural and docking studies. Hence, although our docking studies reveal interesting insights into possible interactions between ligands and the channel protein, which are in line with experimental results, they remain hypothetical until backed up by experimental 3Dstructures.

\section{Conclusions}

We presented a series of structural models for the human voltage-gated sodium channels $\mathrm{Na}_{v} 1.2$ and $\mathrm{Na}_{v} 1.8$ in resting, active, and fast-inactivated state. It was postulated that binding of tetrodotoxin occurred via attractive polar interactions with the selectivity filter residues of domain I and II as well as with the residues of the outer ring. However, sensitivity toward tetrodotoxin depends on the tightness of binding. In sodium channels sensitive to tetrodotoxin block, the aromatic side chains of the Cterminal neighbors of the selectivity filter residues in domains I and II formed a "V"-like structural motif to which the ligand could bind favorably. In toxin-insensitive channel isoforms this "V"-substructure does not exist and binding of tetrodotoxin was less preferred corresponding to experimentally determined binding affinities. Moreover, we developed for the first time a picture of the local anesthetic binding site of voltage-gated sodium channels taking into account all experimental findings concerning local anesthetic block, activation, and interactions with the inactivation gate. We were able to postulate the residues that contribute to the local anesthetic binding site, including those residues which interact only weakly with ligands.

Acknowledgements HS thanks the Swiss Institute of Bioinformatics for support of this work. He also thanks GlaxoSmithKline for generous travel grants and a modified version of SPDBV.

\section{References}

1. Catterall WA (2000) Neuron 26:13-25

2. Hille B (2001) Ion channels of excitable membranes. Sinauer Associates Inc, Sunderland, MA USA

3. Sato C, Ueno Y, Asai K, Takahashi K, Sato M, Engel A, Fujiyoshi Y (2001) Nature 409:1047-1051

4. Ragsdale DS, McPhee JC, Scheuer T, Catterall WA (1994) Science 265:1724-1728

5. Ragsdale DS, McPhee JC, Scheuer T, Catterall WA (1996) Proc Natl Acad Sci USA 93:9270-9275

6. Linford NJ, Cantrell AR, Qu Y, Scheuer T, Catterall WA (1998) Proc Natl Acad Sci USA 95:13947-13952

7. Wang GK, Quan C, Wang S (1998) Pflugers Arch 435:293-302

8. Wright SN, Wang SY, Wang GK (1998) Mol Pharmacol 54:733-739

9. Nau C, Wang SY, Strichartz GR, Wang GK (1999) Mol Pharmacol $56: 404-413$

10. Sunami A, Glaaser IW, Fozzard HA (2000) Proc Natl Acad Sci USA 97:2326-2331
11. Yarov-Yarovoy V, Brown J, Sharp EM, Clare JJ, Scheuer T, Catterall WA (2001) J Biol Chem 276:20-27

12. Yarov-Yarovoy V, McPhee JC, Idsvoog D, Pate C, Scheuer T, Catterall WA (2002) J Biol Chem 277:35393-35401

13. Vassilev PM, Scheuer T, Catterall WA (1988) Science 241:1658-1661

14. Stuhmer W, Conti F, Suzuki H, Wang XD, Noda M, Yahagi N, Kubo H, Numa S (1989) Nature 339:597-603

15. Patton DE, West JW, Catterall WA, Goldin AL (1992) Proc Natl Acad Sci USA 89:10905-10909

16. West JW, Patton DE, Scheuer T, Wang Y, Goldin AL Catterall WA (1992) Proc Natl Acad Sci USA 89:10910-10914

17. Penzotti JL, Fozzard HA, Lipkind GM, Dudley Jr SC (1998) Biophys J 75:2647-2657

18. Lipkind GM, Fozzard HA (1994) Biophys J 66:1-13

19. Guy HR, Durell SR (1996) Developing three-dimensional models of ion channel proteins. In: Narahashi $\mathrm{T}$ (ed) Ion Channels, vol 4. Plenum, New York, London, pp 1-40

20. Guy HR, Durell SR (1995) Structural model of $\mathrm{Na}+, \mathrm{Ca} 2+$, and $\mathrm{K}+$ channels. In: Dawson D (ed) Ion channels and genetic diseases. The Rockefeller University Press, New York, pp 1-16

21. Jiang Y, Lee A, Chen J, Cadene M, Chait BT, MacKinnon R (2002) Nature 417:515-522

22. Jiang Y, Lee A, Chen J, Cadene M, Chait BT, MacKinnon R (2002) Nature 417:523-526

23. Doyle DA, Morais Cabral J, Pfuetzner RA, Kuo A, Gulbis JM, Cohen SL, Chait BT, MacKinnon R (1998) Science 280:69-77

24. Lipkind GM, Fozzard HA (2000) Biochemistry 39:8161-8170

25. Khan A, Romantseva L, Lam A, Lipkind G, Fozzard HA (2002) J Physiol 543:71-84

26. Tikhonov DB, Zhorov BS (2005) Biophys J 88:184-197

27. Guex N, Peitsch MC (1997) Electrophoresis 18:2714-2723

28. Teplyakov A, Obmolova G, Badet-Denisot MA, Badet B, Polikarpov I (1998) Structure 6:1047-1055

29. van Gunsteren WF, Billeter SR, Eising AA, Hünenberger PH, Krüger P, Mark AE, Scott WRP, Tironi IG (1996) Biomolecular Simulation: The GROMOS 96 Manual and User Guide. vdf Hochschulverlag AG an der ETH Zürich, Zürich

30. Rohl CA, Boeckman FA, Baker C, Scheuer T, Catterall WA, Klevit RE (1999) Biochemistry 38:855-861

31. Laskowski RA, MacArthur MW, Moss DS, Thornton JM (1993) J Appl Cryst 26:283-291

32. McMartin C, Bohacek RS (1997) J Comput Aided Mol Des 11:333-344

33. Fozzard HA, Lipkind G (1996) Jpn Heart J 37:683-692

34. Hille B (1971) J Gen Physiol 58:599-619

35. Hille B (1975) Biophys J 15:615-619

36. Guo XT, Uehara A, Ravindran A, Bryant SH, Hall S, Moczydlowski E (1987) Biochemistry 26:7546-7556

37. Noda M, Suzuki H, Numa S, Stuhmer W (1989) FEBS Lett 259:213-216

38. Ravindran A, Moczydlowski E (1989) Biophys J 55:359-365

39. Terlau H, Heinemann SH, Stuhmer W, Pusch M, Conti F, Imoto K, Numa S (1991) FEBS Lett 293:93-96

40. Doyle DD, Guo Y, Lustig SL, Satin J, Rogart RB, Fozzard HA (1993) J Gen Physiol 101:153-182

41. Elliott AA, Elliott JR (1993) J Physiol 463:39-56

42. Gallivan JP, Dougherty DA (1999) Proc Natl Acad Sci USA 96:9459-9464

43. Hille B (1977) J Gen Physiol 69:497-515

44. Vedantham V, Cannon SC (1999) J Gen Physiol 113:7-16

45. Scheuer T (1999) J Gen Physiol 113:3-6

46. Noda M, Ikeda T, Kayano T, Suzuki H, Takeshima H, Kurasaki M, Takahashi H, Numa S (1986) Nature 320:188-192

47. Akopian AN, Sivilotti L, Wood JN (1996) Nature 379:257-262

48. Choudhary G, Yotsu-Yamashita M, Shang L, Yasumoto T, Dudley Jr SC (2003) Biophys J 84:287-294

49. Huheey JE, Keiter EA, Keiter RL (1993) Inorganic chemistry: principles of structure and reactivity. Harper Collins, New York

50. Allen TW, Andersen OS, Roux B (2004) Proc Natl Acad Sci USA 101:117-122 